\title{
Optical Network Control Overlay Using Silicon VOA Arrays
}

\author{
T. E. Darcie, Fellow, IEEE, Peter F. Driessen, Senior Member, IEEE, Michal Osusky, and W. Lin
}

\begin{abstract}
A silicon-based variable optical attenuator (VOA) is used to overlay optical control data on an underlying high-speed optical data channel. We demonstrate experimentally the addition of a $10.7-\mathrm{MHz}(5 \mathrm{~Kb} / \mathrm{s})$ control subcarrier with $<1-\mathrm{dB}$ penalty to a high-speed channel $(500 \mathrm{Mb} / \mathrm{s})$ and quantify the penalty versus control data rate. The modest modulation bandwidth of the VOA enables control subcarriers at frequencies sufficiently high to avoid gain saturation-induced crosstalk in erbium amplifiers, while using the same devices required for channel gain equalization or power control. At OC-192 data rates, we predict that control-channel data rates of $10 \mathrm{~Kb} / \mathrm{s}$ can be tolerated with $<0.5-\mathrm{dB}$ penalty.
\end{abstract}

Index Terms-Dynamic gain equalization (DGE), optical link status, optical network control, optical networks, power control, subcarrier multiplexing, variable optical attenuators (VOAs).

\section{INTRODUCTION}

$\mathbf{O}$ PTICAL-LAYER control techniques have become important for a variety of optical system and network applications. Numerous concepts have been devised to transport control information for channel power equalization, power control, link status management, and more recently, optical packet switching. In each case, a means is devised to overlay relatively low-speed control information onto the optical channel, while minimizing cost and impairment to the underlying data channel.

Approaches that have been explored for adding this control information include the addition of an out-of-band control subcarrier [1], [2], overlay phase modulation [3], orthogonal modulation techniques [4], [5], addition of a separate control wavelength, and control data added below the low-frequency cutoff (modified by line coding) of the data channel [6]. Each of these approaches provides the ability to overlay control data at the expense of cost, complexity, and penalty to the underlying data channel. Subcarrier, phase modulation, orthogonal modulation, and wavelength-division-multiplexing techniques offer high performance (low penalty, high control-channel data rate), but require additional optical and/or radio-frequency (RF) components. Line coding techniques require modification of the line system format used by existing technology, and may introduce crosstalk in erbium-doped fiber amplifiers (EDFAs) if used for low data rates.

Manuscript received June 1, 2004; revised September 2, 2004. This work was supported in part by the Natural Sciences and Engineering Research Council of Canada.

T. E. Darcie, P. F. Driessen, and M. Osusky are with University of Victoria, Victoria, BC, V8W 3P6, Canada (e-mail: tdarcie@ece.uvic.ca; peter@ece.uvic.ca)

W. Lin is with ANDevices, Inc., Fremont, CA 94538 USA (e-mail: wlin@andevices.com).

Digital Object Identifier 10.1109/LPT.2004.839788
High bandwidth control overlays ( $>1 \mathrm{Mb} / \mathrm{s}$ ) are required for optical packet-switched networks which rely on optical labeling of each packet to provide the required decision information at each control point, e.g., [1]. The additional complexity of required high-bandwidth overlays is an obstacle. Low bandwidth overlays $(<1 \mathrm{Mb} / \mathrm{s})$ are adequate for basic functions like dynamic gain equalization (DGE) and dynamic channel-power equalization (DCE) to minimize the degradation caused by such factors as the frequency-dependent gain of EDFAs [7]. These functions have been implemented using simple low-speed and low-cost devices like variable optical attenuators (VOAs). Networks using optical add-drop capability could benefit from an additional channel or link identification function that operates independently of the underlying transport system. This would be particularly useful when channel-specific information is required to span multiple domains of optical transparency.

For transmission (DGE, DCE) and network link identification applications, it is desirable to have a large number of low-cost widely distributed elements. It is, therefore, worthwhile if not essential to consider techniques and single components that are capable of both per-channel power equalization and insertion of link-specific control information. This suggests that the link control information should be applied with a device that controls optical intensity, which in turn suggests the use of subcarriers or modulation below the low-frequency cutoff, rather than more complex alternatives.

In this letter, we demonstrate the use of silicon-based variable-optical attenuators (VOAs) [8] to overlay low-bandwidth control data. Silicon-based VOAs are an attractive means of implementing DGE and DCE, since they offer low power consumption, integration of numerous components into small modules, and low insertion loss. In addition, they exhibit modest modulation bandwidth that provides the capability of direct addition of control information. These devices can be used to superimpose subcarriers directly on the data channel and to simultaneously compensate for faster dynamic gain effects up to the bandwidth of the VOA $(\sim 1 \mathrm{MHz})$.

We demonstrate a $5-\mathrm{Kb} / \mathrm{s}$ control channel transmitted as a frequency-shift-keyed (FSK) subcarrier with a center frequency of $10.7 \mathrm{MHz}$. The control channel can be detected with a low bit-error rate (BER) while inducing a minimal penalty on the high-speed optical channel. We also quantify the penalty induced on the high-speed channel as a function of the information bandwidth of the control channel, showing that a control channel of $10 \mathrm{~Kb} / \mathrm{s}$ could be added to an OC-192 channel with a $0.5-\mathrm{dB}$ power penalty. 


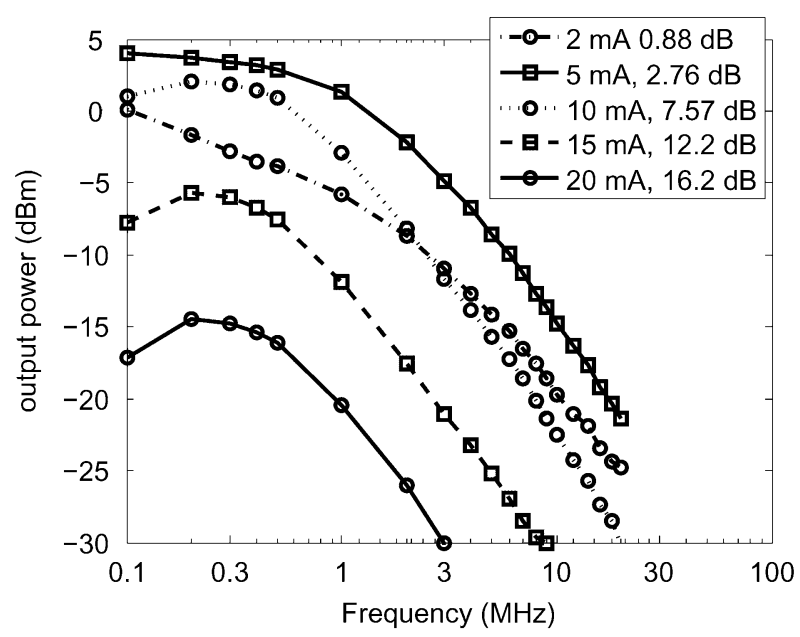

Fig. 1. Small-signal modulation response for VOA as a function of dc bias current and attenuation.

\section{VOA DEVICE CHARACTERIZATION}

The VOA array under test is based on free-carrier absorption in a silicon-on-insulator waveguide [8], manufactured by Lightcross, Inc. Carriers are injected into a laterally defined p-i-n junction, resulting in an optical attenuation coefficient that is proportional to the total carrier density. The device tested is an eight-channel VOA array with a typical fiber-to-fiber insertion loss of $1.5 \mathrm{~dB}$ (zero current). Each VOA had an attenuation of $3 / 10 \mathrm{~dB}$ at currents of 5.3/12.3 mA.

The modulation response of each VOA was measured, as shown in Fig. 1, for $1.3 \mu \mathrm{m}$ (identical $1.55 \mu \mathrm{m}$ ). To avoid RF pickup in the receiver at low frequencies, we used an offset-frequency measurement technique [9]. A lithium-niobate external modulator was used to modulate the input optical signal at an offset frequency (typically between 40 and $100 \mathrm{MHz}$ ). A second signal generator was used to modulate the VOA. The resulting optical-intensity mixing created sum and difference frequencies in the RF intensity domain. The $0.1-16-\mathrm{MHz}$ frequency response of the VOA was then measured as the response of the sum or difference product. For a current of $2 \mathrm{~mA}$, the $3-\mathrm{dB}$ bandwidth is approximately $300 \mathrm{KHz}$. While this bandwidth could be increased by alternative device design parameters, it is adequate for the target applications. Since the modulation response depends on dc bias, some adjustment of modulation current would be required to maintain a constant control-signal amplitude, if our technique were used in conjunction with another power equalization technique.

\section{Control Channel Implementation}

To demonstrate the use of the VOA to add control information, we tested the VOA on a $500-\mathrm{Mb} / \mathrm{s}$ fiber link, as shown in Fig. 2. Either the 1.3- or $1.55-\mu \mathrm{m}$ laser source was coupled through a polarization controller (PC) and the lithium-niobate modulator and modulated with a pseudorandom pattern of length $2^{23}-1$. The avalanche photodiode receiver used transimpedance preamplifier with a $2.5-\mathrm{GHz}$ bandwidth. The frequency modulator $(\mathrm{FM})$ was operated at $5 \mathrm{~Kb} / \mathrm{s}$ with a frequency deviation of $4 \mathrm{KHz}$ and a $2^{15}-1$ pseudorandom pattern. The

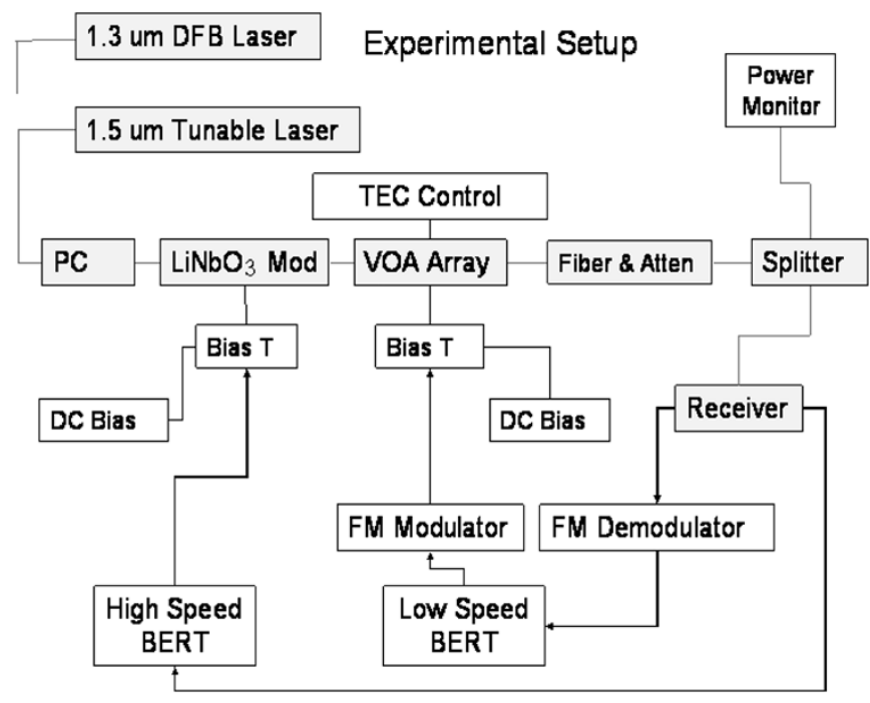

Fig. 2. Experimental setup for VOA control-channel insertion.

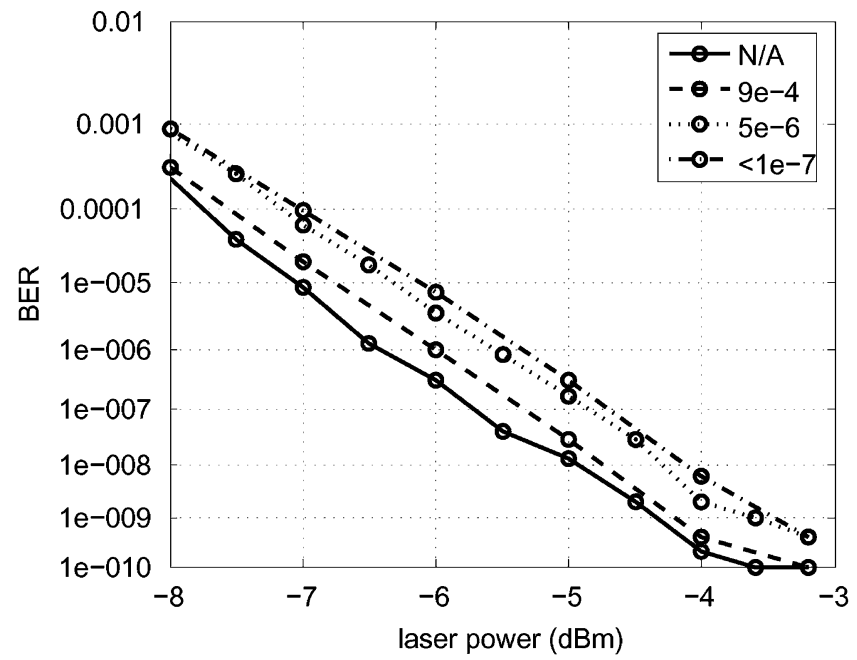

Fig. 3. Measured BER for 500-Mb/s channel for different values of control-channel modulation voltage, and the corresponding control-channel error rate.

RF frequency of $10.7 \mathrm{MHz}$ conformed to a standard analog FM-radio intermediate frequency. The narrow-band control subcarrier requires only a small modulation depth for error-free detection. Therefore, despite the limited frequency response of the VOA at $10.7 \mathrm{MHz}(-20 \mathrm{~dB}$ relative to $100 \mathrm{KHz})$, drive levels for the VOA were compatible with typical FM modulator output levels $(200 \mathrm{mV})$.

\section{RESULTS AND ANALYSIS}

Fig. 3 shows the measured BER for the high-speed channel at $1.5 \mu \mathrm{m}$ for four different levels of control-channel modulation voltage. The tradeoff between the BER of the control channel and the data channel results in an error-free $\left(<10^{-7}\right)$ control channel with roughly a $1-\mathrm{dB}$ penalty to the data channel. Penalties less than $1 \mathrm{~dB}$ to the data channel would be possible with an error-free control channel if 1) error-correction codes were used on the control channel, 2) the control-channel bit rate was 
reduced slightly, and/or 3) the bit rate of the data channel was increased.

To explore this tradeoff, consider the signal-to-interference ratio (SIR) and the resulting BER of a system with two data streams superimposed on a channel: amplitude shift keying modulation at rate $1 / T_{1}=500 \mathrm{Mb} / \mathrm{s}$ with bandwidth $B_{1}=1 / T_{1}$ and average power $P_{1}$, overlaid with FSK modulation at rate $1 / T_{2}=5 \mathrm{~Kb} / \mathrm{s}$ with deviation $f_{d}=4 \mathrm{KHz}$, power $P_{2}$ and bandwidth $B_{2}=1 / T_{2}+2 f_{d}$ (from Carson's rule for FM). Given the power-spectral densities $E_{1}=P_{1} / B_{1}$ and $E_{2}=P_{2} / B_{2}$, assumed constant across the channels

$$
\begin{aligned}
& \mathrm{SIR}_{1}=P_{1} / I_{2}=E_{1} B_{1} /\left(E_{2} B_{2}\right) \\
& \mathrm{SIR}_{2}=P_{2} / I_{1}=E_{2} B_{2} /\left(E_{1} B_{2}\right) .
\end{aligned}
$$

The maximum rate on the control channel is defined by the data rate and the required SIR for each channel, as can be seen by combining (1) and (2) to obtain $\mathrm{SIR}_{1} \mathrm{SIR}_{2}=B_{1} / B_{2}$. For our experimental parameters

$$
\mathrm{SIR}_{1} \mathrm{SIR}_{2}=B_{1} / B_{2}=500 \mathrm{M} / 13 K=3.8 \cdot 10^{4} .
$$

The power penalty incurred on the high-speed channel due to interference from the control channel is

$$
\Delta=\frac{P_{1} / N_{1}}{P_{1} /\left(N_{1}+I_{2}\right)}=1+\frac{I_{2}}{N_{1}}=1+\frac{E_{2} B_{2}}{N_{o} B_{1}}
$$

where $N_{1}$ is the total noise power and $N_{0}$ the noise spectral density. Assuming $\mathrm{SIR}_{1}=\mathrm{SIR}_{2}$, then from (1), (2)

$$
E_{2} / E_{1}=\sqrt{B_{1} / B_{2}}
$$

and from (4)

$$
\Delta=1+\left(E_{1} / N_{0}\right) \sqrt{B_{2} / B_{1}} .
$$

For our experimental results with a BER of $10^{-9}, E_{1} / N_{o}=$ $P_{1} / N_{1}=40$ resulting in $\Delta=0.8 \mathrm{~dB}$. The agreement with the measured penalty of approximately $1 \mathrm{~dB}$ is respectable, given that we have assumed constant power-spectral density across both data and control channels.

This simple analysis results in the dependencies of the datachannel bit rate $B_{1}$, control-channel bit rate $B_{2}$, and controlchannel-induced penalty $\Delta$ shown in Fig. 4 . At OC-192 data rates, we anticipate that control-channel data rates of $10 \mathrm{~Kb} / \mathrm{s}$ can be tolerated with a $0.5-\mathrm{dB}$ penalty (from Fig. 4 and multiplied by a factor of two to be consistent with experimental results).

Having written control information on a high-speed channel, far greater utility could be realized if this could be erased and rewritten at subsequent network control nodes. This has been demonstrated [6] by detecting the incoming control subcarrier and using a simple feed-forward circuit to eliminate the incoming modulation.

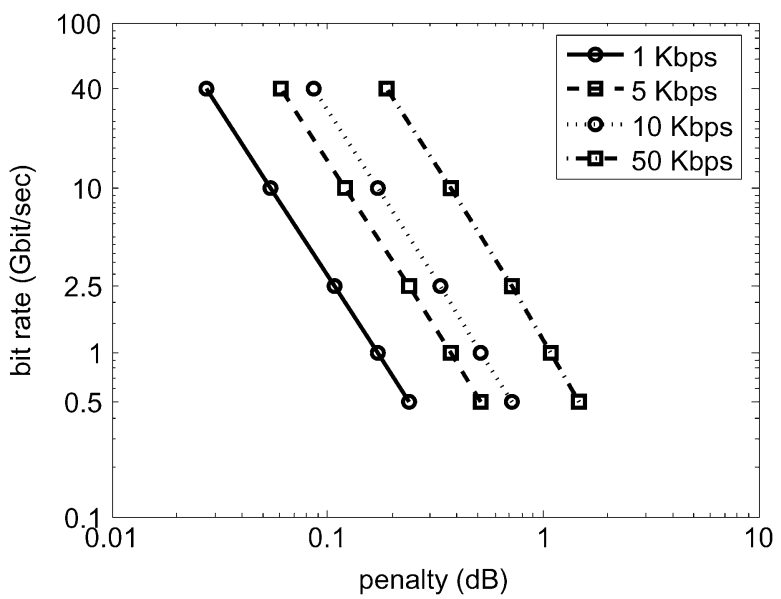

Fig. 4. Predicted penalty to high-speed channel versus bit rate, for given bit rate of control channel.

\section{CONCLUSION}

We have demonstrated the addition (by direct over-modulation) of control information on a high-speed optical channel using a silicon VOA and quantified the tolerable control-channel data rate for any high-speed channel bit rate. By adding the control information as a 10.7-MHz subcarrier, we are able to avoid crosstalk anticipated from the combination of lower speed modulators and EDFA gain dynamics. Combining single-channel gain equalization with optical-layer control information in the same low-cost integrated module (potentially with other passive and active components) may provide a valuable capability for future networks.

\section{REFERENCES}

[1] E. Willner, "Implementing subcarrier-based control in optical networking," in 2002 IEEE MTT-S Dig., New York, 2002, pp. 1687-1690.

[2] R. Gaudino and D. J. Blumenthal, "Remote provisioning of a reconfigurable WDM multichannel add/drop multiplexer," IEEE Photon. Technol. Lett., vol. 11, no. 8, pp. 1060-1062, Aug. 1999.

[3] M. Tomizawa, "Phase-modulation-shift-keying for fast dispersion compensation and optical labeling in optical packet switched networks," in Opt. Fiber Commun. Conf. 2004, Los Angeles, CA, 2004, Paper WF4.

[4] N. Chi, J. Zhang, P. V. Holm-Nielsen, C. Peucheret, and P. Jeppesen "Transmission and transparent wavelength conversion of an optically labeled signal using ASK/DPSK orthogonal modulation," IEEE Photon. Technol. Lett., vol. 15, no. 5, pp. 760-762, May 2003.

[5] J. Zhang, P. V. Holm-Nielsen, N. Chi, C. Peucheret, and P. Jeppesen, "DC-balanced line encoding for optical labeling scheme using orthogonal modulation," in Opt. Fiber Commun. Conf. 2004, Los Angeles, CA, 2004, Paper WF2.

[6] Y. M. Min, M. C. Yuang, S.-L. Lee, and W. I. Way, "Superimposed ASK label in a 10 Gbps multi-hop all-optical label swapping system," in Opt. Fiber Commun. Conf. 2004, Los Angeles, CA, 2004, Paper WF3.

[7] W. J. Tomlinson, "Technologies for dynamic gain and channel power equalization," in Opt. Fiber Commun. Conf. 2003, vol. 1. Washington, D.C., 2003, Paper TuM1, pp. 244-246.

[8] A. Vonsovici, I. E. Day, A. House, and M. Asghari, "ASOC multichannel electronic variable optical attenuator," in Photonics West 2001, Proc. SPIE, vol. 4293, 2001, pp. 1-9.

[9] T. E. Darcie, S. O'Brien, G. Raybon, and C. A. Burrus, "Optical mixerpreamplifier for lightwave subcarrier systems," Electron. Lett., vol. 24, pp. 179-180, 1988. 